both types. Similarly, subculture of broth cultures derived from single colonies of either colonial type, yielded both types of colony on blood agar.

The carbohydrate fermentation and other biochemical tests included in API STREP did not reveal any biochemical differences between the two colonial types of any isolate tested. Furthermore, using a standard controlled disc-diffusion method, we found no differences in antimicrobial susceptibilities between the two colonial types of any isolate tested. Ribotyping did not distinguish between the two colonial types of $E$ faecium E227, an isolate from the bile of a liver transplant recipient.

\section{Discussion}

The explanation for this variation in morphology is not clear. It may be related to the alteration in cell-wall structure that occurs with vancomycin resistance, ${ }^{6}$ as we have yet to see such colonial variation in any van- comycin sensitive isolates of $E$ faecium. This phenomenon may assume particular importance when interpreting culture results. Pure cultures of a vancomycin resistant $E$ faecium from clinical specimens may appear mixed which may detract from the importance of the culture results. A considerable amount of time and effort may also be wasted in attempting to isolate pure cultures when the "contaminant" is, in reality, the second colonial type.

1 Hoffmann SA, Moellering RC. The enterococcus: "putting the bug in our ears". Ann Intern Med 1987;106:757-61.

2 Moellering RC. The enterococcus: a classic example of the impact of antimicrobial resistance on therapeutic the impact of antimicrobial resistance on the $\mathcal{A}$ Antimicrob Chemother 1991;28:1-12.

3 Murray BE. The life and times of the enterococcus. Clin Microbiol Rev 1990;3:46-65.

4 Uttley AHC, Collins CH, Naidoo J, George RC. Vancomycin-resistant enterococci. Lancet 1988;i:57-8.

5 Wade J, Baillie L, Rolando N, Casewell MW Pristinamycin for Enterococcus faecium resistant to vancomycin and gentamicin. Lancet 1992;i:312-3.

6 Courvalin P. Resistance of enterococci to glycopeptides. Antimicrob Agents Chemother 1990;34:2291-6.

\title{
Measurement of serum arabinitol by gas-liquid chromatography: Limitations for detection of systemic candida infections
}

\author{
C McSharry, C Lewis, G Cruickshank, M D Richardson
}

\begin{abstract}
The measurement of serum arabinitol in the diagnosis of systemic candidosis was evaluated using a gas-liquid chromatography technique in a cohort of at risk patients. The prevalence of seropositivity was low and did not correlate with evidence of infection. This technique is unlikely to achieve acceptance because it does not discriminate between patients with and without infection; it requires specialised equipment and it is expensive.
\end{abstract}

(F Clin Pathol 1993;46:475-476)

Department of

Immunology,

University of Glasgow

(Western Infirmary),

Glasgow G11 6NT

Scotland

C McSharry

C Lewis

G Cruickshank

Regional Mycology Reference Laboratory Western Infirmary M D Richardson

Correspondence to: Dr C McSharry

Accepted for publication

17 November 1992 procedures for the rapid testing for deep seated candidosis. D-arabinitol is a major metabolite of the clinically important Candida species, and previous work has suggested that measurement of this pentitol in serum by gasliquid chromatography (GLC) is rapid, specific, and suitable for use in the diagnostic laboratory of a larger general hospital. ${ }^{12}$ To examine this claim we undertook a retrospective assessment (1987-90) of 246 measurements of serum arabinitol in 213 patients.
Methods

All the patients were potentially immunocompromised. Most had leukaemia or were from intensive therapy units, and had a fever of unknown origin. Blood samples were sent from 213 adults and 65 children, 22 of whom were neonates. Thirty three patients had repeat sera taken within 10 days of the original specimen.

Serum arabinitol was measured by a GLC technique. ${ }^{1}$ Briefly, this entailed removal of serum protein (usually $200 \mu \mathrm{l}$ ) with 2 volumes of acetone containing a standard amount of mannitol. The supernatant fluid was dried by evaporation and reconstituted in a reagent mix which sililated the sugars and sugar-alcohols (Sylon HTP kit containing hexamethyldisilazane, trimethylcholorosilane, and pyridine; Supelco Inc., Bellefonte Philadelphia, USA) which rendered them volatile. GLC apparatus (Sigma 3 gas chromatograph, Perkin-Elmer Ltd, Bucks, England). The arabinitol peak was identified by its relative mobility compared with the peak corresponding to mannitol, and was quantified by their relative heights. The quality control of the procedure was assessed by the satisfactory 
measurement of arabinitol in normal serum spiked with known amounts of D-arabinitol.

Candida mannan antigenaemia was measured using a latex-antigen agglutination test kit which was capable of detecting as little as $25 \mathrm{ng}$ mannan/ml serum (CandTec, Ramco Labs, Houston, Texas, USA), and serum anti-Candida precipitating antibodies were demonstrated by countercurrent immunoelectrophoresis (CIE).

\section{Results and discussion}

Only eight patients in this highly selected group had arabinitol concentrations (mean $1.4 \mu \mathrm{g} / \mathrm{ml}$ ) significantly greater than the normal range; $0-1 \cdot 1 \mu \mathrm{g} / \mathrm{ml} .{ }^{1}$ There was no correlation between the arabinitol concentration and subsequent confirmation of infection by either culture, histological examination, or Candida mannan antigenaemia. This agrees with previously presented but unpublished results from our laboratory, before 1987 (Deacon AG, Richardson MD, abstract presented at Proceedings of the First International Symposium on Fungal Antigens; Institute Pasteur, Paris, November 17-20, 1986). These compared the evidence for Candida infection by using the measurement of antigen, arabinitol, and anti-Candida precipitating antibodies in a retrospective study of 40 sera from 11 patients, six confirmed and five probable cases of invasive candidosis, and a prospective examination of 89 sera from 31 adults and 22 children. None of the 11 patients from the retrospective study had a positive antigen reaction, although all had at least one raised arabinitol value and six had positive CIE precipitins. In the prospective series 11 of the 89 patients had positive antigen tests but none had raised arabinitol concentrations and none was independently confirmed to have systemic infection.

Of the 33 repeat sera in the present study, the 18 with the lowest initial arabinitol concentrations remained low. In the other 15 with the higher concentrations, who had been started on prophylactic antifungal treatment since the original sample, there was a signifi-
Median and interquartile range for serum arabinitol concentrations

\begin{tabular}{lll}
\hline Group & Median & Interquartile range \\
\hline All $(\mathrm{n}=213)$ & 0.3 & $0 \cdot 18-0.53$ \\
Adults $(\mathrm{n}=148)$ & 0.28 & $0.17-0.47$ \\
Children $(\mathrm{n}=65)$ & 0.4 & $0.21-0.71$ \\
Neonates $(\mathrm{n}=22)$ & 0.57 & $0.31-0.75$ \\
\hline
\end{tabular}

cant decrease in the arabinitol concentrations, means (plus SD), respectively, $1.05 \mu \mathrm{g} / \mathrm{ml}$ $(0.7)$ and $0.51 \quad(0.4) \mu \mathrm{g} / \mathrm{ml}$ (two-sample $t$-test, $t=2.57, \mathrm{p}=0.018)$. Any potential clinical value of this observation is undermined by the fact that these concentrations were largely within the normal range.

We found that there was no significant difference between males or females in the present study group but there was a significantly greater concentration of arabinitol in children, especially neonates, than in adults; Mann-Whitney U-test, $p=0.005$ (table).

In our experience the GLC test for serum arabinitol is of little routine value for the following reasons. First, it does not sufficiently discriminate between patients with and without infection; secondly, it requires specialised equipment and is expensive; around $£ 42.66$ for each serum sample. Despite the highly selected risk group tested there were very few positive tests, and even with these it was difficult to be confident without independent confirmation by positive culture or histological analysis. When reporting the arabinitol results, usually within 2 hours of receipt of what was generally an urgent request, we found that most clinicians had already started anti-fungal treatment in at risk patients irrespective of arabinitol concentration. The observation that children, especially neonates, had significantly higher arabinitol values than adults may be of some biochemical importance.

1 Deacon AG. Estimations of serum arabinitol for diagnosing invasive candidosis. I Clin Pathol 1986;39:842-50.

2 Eng RHK, Chmel H, Buse M. Serum levels of arabinito in the detection of invasive Candidiasis in animals and humans. F Infect Dis 1981;143:677-83. 